\title{
Sintomatología depresiva, ideación suicida y autoconcepto en una muestra de niños mexicanos
}

\author{
Depressive Symptomatology, Suicidal Ideation and Self-concept in a Sample \\ of mexican Children \\ Sintomatologia depressiva, ideação suicida e autoconceito em uma amostra \\ de crianças mexicanas
}

\author{
Ana María Chávez-Hernández*, Fredi Everardo Correa-Romero, \\ Alejandro León Klein-Caballero, Luis Fernando Macías-García \\ Universidad de Guanajuato \\ Karen Viviana Cardoso-Espindola, Irma Beatríz Acosta-Rojas \\ Círculo de Estudios de Psicología Profunda
}

Doi: http://dx.doi.org/10.12804/revistas.urosario.edu.co/apl/a.4733

\section{Resumen}

Diversas investigaciones demuestran el incremento de la depresión infantil en varios países, y apuntan al autoconcepto como predictor asociado con la depresión, riesgo suicida y otras conductas de riesgo que pueden manifestarse en la infancia o posteriormente. Existen escasos estudios publicados en México sobre depresión y comportamiento suicida en niños, a pesar del alarmante aumento del suicidio consumado en este grupo etario. Por ello, este estudio tuvo por objetivo describir la relación entre depresión y autoconcepto en una muestra de niños escolarizados del estado de Guanajuato, México. El presente fue un estudio descriptivo, exposfacto, transversal, con un muestreo intencional; participaron 217 niños escolarizados de ambos sexos y entre 9 y 13 años de edad. Se aplicó el Inventario de depresión infantil y el Cuestionario de autoconcepto general. Los resultados mostraron puntajes por encima del esperado en depresión leve/moderada y severa, ideación suicida y bajo autoconcepto. Se encontraron niveles de correlación significativos entre el autoconcepto, la sintomatología depresiva y el sexo. Se sugieren estrategias psicoeducativas que favorezcan la temprana detección y el adecuado abordaje de los signos de sufrimiento emocional y conductas de riesgo en niños. Palabras clave: depresión, autoconcepto, autoestima, niños, ideación suicida, México.

\section{Abstract}

Previous studies have shown the prevalence of depression in children, and have suggested that self-concept as the key variable to predict depressive symptoms,

* Universidad de Guanajuato. Correspondencia: Ana-María Chávez-Hernández, correo electrónico: anachavez@ugto.mx

Cómo citar este artículo: Chávez-Hernández, A. M., Correa-Romero, F. E., Klein-Caballero, A. L., Macías-García, L. F., Cardoso-Espindola, K. V., \& Acosta-Rojas, I. B. (2017). Sintomatología depresiva, ideación suicida y autoconcepto en una muestra de niños mexicanos. Avances en Psicología Latinoamericana, 35(3), 501-514. doi: http://dx.doi.org/10.12804/revistas. urosario.edu.co/apl/a.4733 
suicide risk and other risk behaviors, that may emerge during childhood or later in life. In Mexico, there are few reported studies about depression or suicidal ideation in children, despite the increasing suicide rate in this age group. This study was aimed to describe the relationship among depression and self-concept in a sample of children enrolled in primary schools of Guanajuato State, Mexico. This was a descriptive, transversal and ex post facto study with a non-probabilistic sample of 217 children of both sexes and 9-13 years old. The instruments used were: Child Depression Inventory and the Questionnaire of General Self Concept. The results of this research showed higher levels of mild/ moderate and severe Depression, Suicidal ideation, and low Self-concept. A significant correlation was found between Self-concept factors and Depressive Symptoms, and with sex. Psycho-educative strategies are suggested to appropriately detect and adequately address signs of depression, emotional suffering and risk behaviors in children.

Keywords: Depression, self-concept, self-esteem, children, suicidal ideation, Mexico.

\section{Resumo}

Diversas pesquisas demostram o incremento da Depressão infantil em vários países, e apontam ao autoconceito como preditor associado da Depressão, risco suicida e outras condutas de risco, que podem manifestar-se na infância ou posteriormente. Existem escassos estudos publicados no México sobre Depressão e comportamento suicida em crianças, a pesar do alarmante aumento do suicídio consumado neste grupo etário. Este estudo teve por objetivo descrever a relação entre Depressão e autoconceito em uma amostra de crianças escolarizadas do estado de Guanajuato, México. O presente foi um estudo descritivo, ex-post-facto, transversal, com uma amostragem intencional; participaram 217 crianças escolarizadas, ambos os sexos, entre 9 e 13 anos de idade. Aplicaram-se o Inventário de Depressão Infantil e o Questionário de Autoconceito Geral. Os resultados mostraram pontuações por cima do esperado em Depressão leve/moderada e severa, Ideação
Suicida e baixo autoconceito. Se encontraram níveis de correlação significativos entre o Autoconceito e a sintomatologia depressiva e o sexo. Sugerem-se estratégias psico-educativas que favoreçam a prematura detecção e adequada abordagem dos signos de sofrimento emocional e condutas de risco em crianças.

Palavras-chave: depressão, autoconceito, autoestima, crianças, ideação Suicida, México.

\section{Introducción}

La depresión infantil (DI) es un tema de interés relativamente reciente debido a que solía considerarse que la depresión era un problema que esencialmente afectaba a los adultos. Actualmente, se reconoce la relevancia de su estudio debido a las consecuencias negativas para los niños en las esferas sociales, educativas, físicas y mentales. Las secuelas de la DI mal diagnosticada o atendida pueden presentarse a mediano o largo plazo, pues siendo la infancia la etapa que antecede a la adolescencia, existe alta probabilidad de que en este periodo de cambio se manifiesten conductas de riesgo tales como alcoholismo, abuso de sustancias, ideación y conductas suicidas, entre otras (Acosta-Hernández et al., 2011).

La depresión se caracteriza principalmente por largos periodos de tristeza que afectan el pensamiento y el comportamiento de las personas, provoca pérdida de interés en las actividades, afecta los hábitos alimenticios, de sueño, lleva a la incapacidad de experimentar placer o divertirse y deteriora las relaciones sociales de quienes se ven afectados por ella (Asociación Americana de Psiquiatría - APA - 2014). De acuerdo con Vogel(2012), en el mundo occidental la DI (situada entre los 6 a los 12 años) se presenta en el $1 \%$ y $2 \%$ de la población, mientras que la depresión adolescente (edades 13 a 18 años) se presenta del $4 \%$ al $8 \%$ de la población general. Sin embargo, existen estudios que han obtenido datos superiores al $30 \%$ de prevalencia de riesgo y síntomas 
depresivos en niños en edades escolares (Gallegos, Linan-Thompson, Stark \& Ruvalcaba, 2013; Rodríguez-Espínola, 2010).

La DI suele presentarse en conjunto con desórdenes emocionales y de ansiedad (Maughan, Collishaw \& Stringaris, 2013). Los síntomas depresivos en la infancia pueden ser similares a los observados en la adultez, pero algunas diferencias importantes en los niños incluyen dificultades en las relaciones con su grupo de pares y en su autoconcepto, además se ha encontrado que padecer depresión en la infancia es un predictor para su presencia en la vida adulta (McCabe, Ricciardelli \& Banfield, 2011).

\section{Algunos estudios sobre depresión infantil}

En Estados Unidos se llevó a cabo una investigación con 2351 niñas de 7 años de edad y sus cuidadores, con la finalidad de analizar durante un periodo de 6 años la correlación entre la parentalidad y el comportamiento observado en las menores. De acuerdo con los resultados, los castigos severos y la falta de afecto fueron los elementos que influyeron de forma significativa en la presencia de conducta depresiva de las niñas (Hipwell et al., 2008).

Por otra parte, diversos estudios resaltan la importancia de los contextos no familiares en la DI, particularmente los contextos escolares. En este sentido, Bernaras, Jaureguizar, Soroa, Ibabe y Cuevas (2011) analizaron la depresión en 1102 niños entre 8 y 12 años de edad en el País Vasco (España) y encontraron síntomas de depresión significativa en el 4,3\% de la muestra, al mismo tiempo que observaron que la depresión se predecía por la desadaptación escolar, lo mismo que la baja autoestima.

Aunado a lo anterior, Esparza y Rodríguez (2009) realizaron una investigación con la finalidad de identificar los factores contextuales relacionados con los estados de ansiedad y depresión en niños escolarizados de Colombia; la muestra estuvo constituida por 254 menores entre 6 y 11 años de edad y sus resultados reportaron que las relaciones sociales y exigencias académicas se asociaron con los estados de depresión en dicha población.

Otro estudio realizado en la ciudad de Bogotá, Colombia (Pérez-Olmos, Téllez, Vélez \& Ibáñez-Pinilla, 2012) tuvo por objetivo analizar la relación entre riesgo suicida y enfermedad mental en 309 estudiantes del nivel secundaria, con edad promedio de 13,8 años; los resultados presentaron un alto puntaje de probabilidad de riesgo para el comportamiento suicida y síntomas mentales (47,6\%), mientras que el $26,5 \%$ de la muestra manifestó riesgo suicida; ambas variables se explicaron conjuntamente por la depresión, baja autoestima, disfunción familiar severa, sexo femenino y la edad mayor o igual a 15 años. EI maltrato psicológico seguido del abuso físico se asociaron con manifestación suicida y/o enfermedad mental, y la buena relación familiar, con menor probabilidad de presencia de estos riesgos.

Las relaciones de pares entre niños de grado escolar también deben considerarse en el estudio del desarrollo de síntomas depresivos, las cuales implican el desarrollo adaptativo, la aceptación y la amistad o, por el otro lado, el desarrollo desadaptativo, el rechazo de pares y las dificultades para establecer relaciones. Un factor que modula sobresalientemente las relaciones de pares en la edad infantil es la agresión, y ese fue el punto de partida de la investigación de Chen, Huang, Wang y Chang (2012), quienes encontraron que los niños agresivos, aunque puedan ser populares en los grupos, suelen ser más solitarios debido a que forman menos relaciones cercanas y significativas; por lo tanto, la agresión contribuye negativamente a las relaciones de pares y, en consecuencia, favorece la presencia de depresión.

Cohen, Young y Abela (2012) buscaron los estilos cognoscitivos que se relacionaban con la depresión en 206 niños y encontraron que aquellos que se evaluaban a sí mismos de forma más 
negativa, eran más propensos y vulnerables a la depresión que aquellos que se evaluaban más positivamente.

\section{Estudios sobre autoconcepto en niños}

Por otro lado, se sabe que para que los niños puedan desarrollarse adecuadamente son necesarias las relaciones emocionales cercanas que promuevan un autoconcepto positivo, así como la estimulación cognitiva y psicosocial. El autoconcepto (AC) se refiere a la organización interna de actitudes, sentimientos, expectativas y significados de sí, que orientan la interpretación y la organización de las experiencias y acciones de los niños (Harter, 2006, citado en Inguglia, Ingoglia \& LoCoco, 2013). Un adecuado AC ayuda a los niños a entender su ambiente y les permite reaccionar adecuadamente al contexto, influye en la adaptación social y promueve el bienestar, el desempeño académico y las relaciones sociales positivas.

El ambiente familiar es importante para que los niños puedan desarrollar un AC positivo, pues la interacción con los integrantes de su familia les permite crear juicios y evaluaciones sobre sí. Un estudio realizado en Turquía (Yazici \& Tastepe, 2013) concluyó que el AC y la autoestima de los niños se afectaba positivamente cuando las familias tenían convivencia de unidad y compañía, en comparación con las familias de convivencia de control y adversidad. En el mismo sentido, se ha encontrado que el maltrato infantil, el desinterés por el cuidado, la negligencia y el excesivo uso de la disciplina severa, dañan el AC y favorecen la aparición de depresión (Turner, Finkelhor \& Ormrod, 2010).

En una investigación realizada en Australia con 510 niños entre 7 y 13 años de edad, el $23 \%$ reportó altos niveles de depresión clínica, comparándolos con aquellos niños que presentaron niveles normales de síntomas depresivos (McCabe et al., 2011). Se encontró que los del primer grupo obtuvieron puntajes más altos en los afectos negativos, menor autoestima y AC más negativo en contraste con los del segundo grupo. Además, el estudio mostró que a mayores puntajes de $\mathrm{AC}$ negativo mayor fuerza de la asociación entre depresión, y también en cuanto las expectativas de los niños estuvieran relacionadas con sensación de fracaso y menor rendimiento personal, existía ansiedad y riesgo suicida. Otros efectos encontrados del AC negativo fueron menores puntuaciones en actitudes prosociales, un funcionamiento social deteriorado y menor amistad entre pares.

En su estudio realizado en el País Vasco (España), Garaigordobil, Pérez y Mozaz (2008) señalan que el AC negativo no se relacionó únicamente con la sintomatología depresiva, sino también encontraron alta relación con problemas psicosomáticos, conductas obsesivo-compulsivas, dificultades de socialización, depresión y hostilidad.

Molina y Raimundi (2011) realizaron una investigación en Argentina con la finalidad de analizar la influencia de las autopercepciones específicas sobre la autoestima global en 219 niños de escuela primaria. De acuerdo con los resultados, se encontró como el principal predictor de autoestima global en las niñas la aceptación por parte de los compañeros; mientras que para los varones dicho predictor se relacionó con la autopercepción de su aspecto físico.

\section{Estudios sobre depresión y conducta suicida en niños de la República Mexicana}

En México, Torre, Cubillas, Román y Valdez (2009) realizaron un estudio con la finalidad de analizar e identificar los factores psicológicos asociados con la ideación suicida en niños; seleccionaron una muestra de 631 estudiantes con un rango de edad de 10 a 13 años, quienes se encontraban cursando el quinto y sexto grado de primaria. En dicha investigación, se utilizó el Inventario de Depresión Infantil (CDI), la Escala de Ansiedad Manifiesta en Niños (CMAS-R) y el Inventario de Autoestima de 
Coopersmith. Se encontró que el grupo de niños con ideación suicida $(29,2 \%)$ presentó mayores estados depresivos, así como mayor frecuencia de casos con baja autoestima en comparación con el grupo sin ideación.

Un estudio reciente realizado por Miranda de la Torre, Laborín-Álvarez, Chávez-Hernández, Sandoval y Torres (2016) en 557 niños escolarizados de ambos sexos, con una edad promedio de 10,6 años, de la ciudad de Hermosillo, México, con el fin de obtener las propiedades psicométricas del CDI y evaluar su adecuación y confiabilidad para población mexicana, mostró, entre otros puntos, un alto índice de rasgos depresivos (moderados a severos), sin diferencias significativas en la ocurrencia de la depresión por sexo; asimismo, se concluyó que la escala resulta adecuada y confiable para la población de niños mexicanos.

Por otra parte, Gallegos et al. (2013) estudiaron la efectividad de una intervención cognitiva de promoción de la salud mental realizada en México, la cual consistió en enseñar habilidades sociales, emocionales y estrategias de afrontamiento al estrés a 1030 niños y niñas de escolaridad primaria, y notificaron resultados favorables en la reducción de síntomas depresivos.

Al igual que en muchos países, en México, diversos informes públicos (Instituto Nacional de Estadística y Geografía - INEGI-, 2015a; Instituto Nacional de Salud Pública — INSP_- 2012; Organización Mundial de la Salud — OMS—, 2014) expresan un alarmante incremento de problemáticas psicosociales de la población infantil y juvenil que se manifiesta en conductas de riesgo y trastornos afectivos y del comportamiento que impactan en todas las esferas y ámbitos de acción de los menores de edad.

En México, la Secretaría de Salud (SSA, 2006) calcula que un $2,5 \%$ de la población infantil y un $8,3 \%$ de los adolescentes podría sufrir depresión. Por otra parte, en la Encuesta Nacional de los Hogares elaborada por el INEGI (2014), más de 100 mil menores de entre 7 y 14 años de edad informaron sentirse deprimidos diariamente, de los cuales, 42486 informaron que dicho sentimiento se presenta de forma severa e intensa. Asimismo, de acuerdo con los datos recabados, se encontró que 34120 menores tomaban antidepresivos al momento de la encuesta.

Según un estudio realizado por la OMS (2000), la República Mexicana mostró el porcentaje más alto de incremento de suicidios $(61,9 \%)$ entre todos los países evaluados. Dicho estudio, en el caso de México, evaluó la tasa de suicidio del periodo 1982-1983 que se comparó con la tasa de suicidios del periodo 1993-1995, de esta comparación se obtuvo el primer lugar según el porcentaje de aceleración, específicamente en los grupos de niños y jóvenes. Este incremento se mostró en el periodo de 1990-2000, en un 150\% para los niños mexicanos entre 5 y 14 años de edad y en un $74 \%$ para el grupo entre 15 y 24 años (Bridge, Goldstein \& Brent, 2007, citado en Borges, Benjet, Medina-Mora, Orozco \& Nock, 2008).

Aproximadamente, desde la década de los años de 1990 y hasta la fecha, en México, el grupo de mayor riesgo para consumar el suicidio es entre 15 y 24 años de edad, pero también el suicidio en el grupo de niños de 10 a 14 años de edad ha mostrado un constante y alarmante incremento (INEGI, 2015b). Considerando que a partir de los 15 años se presenta la consumación del suicidio, es esperable encontrar conductas de riesgo suicida, como son la ideación y tentativas de suicidio, desde los años previos, es decir, cuando los niños están cursando los últimos años del nivel básico (Borges, Orozco, Benjet \& Medina-Mora, 2010; Roberts \& Chen, 1995; Páramo-Castillo \& Chávez-Hernández, 2007).

La identificación temprana y el tratamiento de la DI puede reducir el impacto familiar, académico y social, así como disminuir el riesgo de suicidio, abuso de sustancias y trastornos depresivos persistentes en la adultez.

Considerando la relación entre las variables de depresión infantil y autoconcepto, derivada 
de las investigaciones realizadas en varios países, así como la reconocida relación entre depresión e ideación suicida y, además, habiendo escasos estudios en México que aborden la sintomatología depresiva en población infantil, los objetivos del presente estudio fueron: (i) analizar la presencia de sintomatología depresiva e ideación suicida y (ii) conocer su grado de asociación estadística con el autoconcepto en una muestra de niños escolarizados del Estado de Guanajuato, México.

\section{Materiales y métodos}

El presente fue un estudio correlacional con un diseño de tipo exposfacto y transversal.

Participantes. Se realizó un muestreo de tipo intencional no probabilístico de niños escolarizados; el total de participantes fue de 217 niños de 3 escuelas de nivel básico del estado de Guanajuato, México. Los criterios de inclusión fueron que los niños supieran leer, estudiaran los últimos grados de la primaria y que aceptaran libremente participar en el estudio. Con relación al sexo, el 54,8\% fueron varones y 45,2\% mujeres, en un rango de edad entre 9 a 13 años, con un promedio de 11,16 años y con una desviación estándar de 0,7 años. Cabe aclarar que solamente 3 niños tenían 13 años recién, y 6 niños tenían 9 años de edad; el 7,8\% de la muestra estaba cursando el cuarto año, el $43,8 \%$ el quinto año y el 48,4\% el sexto (último) año de primaria.

Instrumentos. Se aplicaron dos instrumentos: (i) Inventario de depresión infantil (CDI) realizado por Kovacs (1992) en idioma inglés, para el presente estudio se utilizó la adaptación al idioma español por Del Barrio y Carrasco (2004). Para determinar la confiabilidad del instrumento se realizó la prueba de alfa de Cronbach, que arrojó un valor de 0,80 , en la versión de la adaptación española, el cual es un indicador adecuado de consistencia interna (Del Barrio \& Carrasco, 2004), y en la prueba de estabilidad del instrumento se obtuvo un valor de 0,38 con un intervalo de dos semanas, en muestras no clínicas, dicha puntuación no es alta, pero es común en población infantil no clínica (Kovacs, 2004). El CDI es un auto informe que consta de 27 ítems para medir sintomatología depresiva en niños y otorga las puntuaciones de: sin sintomatología depresiva, sintomatología depresiva leve/moderada y sintomatología depresiva severa. Además, ofrece índices sobre las escalas que constituyen el factor de depresión: disforia, autoestima negativa e ideación suicida.

Para crear categorías con respecto al nivel de depresión, se utilizan puntos de corte, los cuales dependen de los percentiles y corresponden a los siguientes rangos: de 1 a 89 representa un estado sin sintomatología depresiva; de 90 a 95 corresponde a una sintomatología leve/moderada y de 96 a 99 tiene que ver con una sintomatología severa de depresión. De acuerdo con Kovacs (1992), el punto de corte se encuentra a partir de la suma de 19 puntos, desde donde se puede establecer una sintomatología depresiva con un mínimo de falsos negativos $(7,93 \%)$ y falsos positivos $(10,50 \%)$.

(ii) El cuestionario de autoconcepto general (CAG) (García-Torres, 2001) consta de 48 afirmaciones relacionadas con 6 dimensiones: autoconcepto físico, aceptación social, autoconcepto familiar, autoconcepto intelectual, sensación de control y autoevaluación personal. Los puntos de corte para cada categoría se encuentran relacionados con los siguientes rangos: de 5 a 20 por debajo de la norma; de 25 a 75 dentro de la norma y de 80 a 95 por encima de la norma. Para medir la fiabilidad de la prueba, se realizó la prueba de alfa de Cronbach que arrojó un valor de 0,87 , lo cual indica alta consistencia interna (García-Torres, 2001).

Para ambas escalas se consideró que los criterios de validez de los manuales eran adecuados, debido a que no se encontraron evidencias en la literatura sobre las diferencias importantes entre las muestras con las que se realizó el proceso de validación de las escalas y la muestra del estudio; 
obtener evidencias de validez de ambas escalas para la muestra en estudio no se consideró como objetivo de la presente investigación, lo cual representa una de sus limitantes.

Procedimiento y consideraciones éticas. Se estableció contacto con las autoridades administrativas de las escuelas, se les informó sobre los objetivos del estudio y se obtuvo la firma de la carta de consentimiento informado de autoridades y de los padres de familia. Asimismo, al momento de la aplicación, los psicólogos encuestadores estuvieron respondiendo las dudas surgidas en los niños y explicaron los objetivos del estudio e indicaron además, que su participación era voluntaria y que podían retirarse en cualquier momento, sin que tuviera ninguna consecuencia, por tanto, los niños no recibieron presión ni coerción para participar y debieron firmar también su consentimiento. La aplicación se realizó en grupos pequeños de 5 niños con los de menor edad y en grupos de 10 niños con los mayores, para asegurar mejor comprensión de las preguntas. Parte del compromiso ético incluyó entregar un reporte final de resultados a cada institución educativa. Además, en las escuelas se impartieron conferencias para los padres de familia sobre conductas de riesgo en niños.

Análisis estadístico. Para describir la sintomatología depresiva de la muestra se realizaron pruebas de frecuencias para el puntaje total; se obtuvieron porcentajes para variables como sexo y grado. Con la edad, se realizaron pruebas de medida central (promedio y desviación estándar). También se realizó un análisis de tendencia central para los indicadores del CAG. Se describieron porcentajes para las categorías que el manual sugiere (dentro de la norma, por debajo de la norma y por encima de la norma) para cada indicador; este procedimiento descriptivo es un prerrequisito para analizar la relación de las variables. Posteriormente, se utilizaron las variables con puntajes absolutos del CGA y del CDI que tienen un nivel de medición intervalar. Luego de comprobar la normalidad de las variables por medio de la prueba de Kolmogorov - Smirnov, se determinó que ninguna cumplía con una distribución normal. Por otro lado, se realizaron análisis de correlación de Spearman y de Pearson. Finalmente, para determinar la importancia de los factores del CAG sobre el puntaje total de la sintomatología depresiva, se realizó una regresión lineal por medio del método de pasos sucesivos.

\section{Resultados}

Para medir la depresión se utilizó el inventario de depresión infantil (CDI). De acuerdo con el manual se crearon tres categorías: sin sintomatología depresiva, con depresión leve/moderada y depresión severa. A partir de los 19 puntos se consideró la existencia de sintomatología depresiva.

\section{Resultados sobre depresión infantil}

Los resultados indican que el 88,5\% de los niños no presentaron sintomatología, mientras que el 11,5\% si presentó algún grado de depresión. El $36 \%$ de los niños con sintomatología depresiva estaban cursando el quinto año de primaria (11 años de edad). El restante $64 \%$ se encontraban cursando el sexto grado de primaria (mayormente con 12 años de edad). El $48 \%$ de la muestra que presentó sintomatología depresiva eran varones, mientras que el $52 \%$ eran mujeres.

Con relación en la sintomatología de rasgos depresivos, que la prueba permitió detectar en toda la muestra, se encontró que el 17,1\% de los niños refirieron presentar de manera frecuente sentimientos de tristeza (el 10,8\% mencionó estar triste muchas veces y $6,3 \%$ indicó estar siempre triste).

Respecto a la visión favorable que los menores perciben sobre lo que hacen, el 44,8\% de los niños refiere no estar seguro si las cosas que realizan saldrá bien (se incluye el $3 \%$ que opinó que nunca le saldrá nada bien). En cuanto a la seguridad que los 
menores perciben en las actividades que realizan, se observó que el 4,5\% refiere creer que nada de lo que hace está bien, mientras que el $17,2 \%$ percibe que gran parte de lo que hace está mal.

En cuanto a la percepción que los niños refieren acerca de la vivencia de cosas negativas, el 51,1\% informó sentirse preocupado por la ocurrencia de situaciones negativas (se incluye el 7,8\% que respondió estar seguro de que les ocurrirán cosas malas).

Respecto a la sensación de control sobre las vivencias negativas, el $68,2 \%$ de los menores mencionó que generalmente no tienen la culpa de que estas ocurran; asimismo, el 7,5\% refirió sentirse responsable de todo lo malo que le ocurre y el 24,3 \% eligió la opción: 'muchas cosas malas son culpa mía'.

Tabla 1

Resultados sobre sintomatología depresiva según CDI

\begin{tabular}{lcc}
\hline \multicolumn{1}{c}{ Factor depresión } & $\begin{array}{c}\text { Porcen- } \\
\text { taje }\end{array}$ & $\begin{array}{c}\text { Núm. } \\
\text { niños }\end{array}$ \\
\hline Con sintomatología depresiva & 11,5 & 25 \\
Depresión leve/moderada & 7,3 & 16 \\
Depresión severa & 4,2 & 9 \\
Ideación suicida & 16 & 35 \\
Factor disforia & 10,8 & 23 \\
Estar triste muchas veces & 6,3 & 14 \\
$\begin{array}{l}\text { Estar siempre triste } \\
\text { Culpable de todo lo malo que me }\end{array}$ & 7,5 & 16 \\
ocurre & 24,3 & 53 \\
$\begin{array}{l}\text { Muchas cosas malas son culpa mía } \\
\text { Factor autoestima negativa }\end{array}$ & & \\
No estar seguro de hacer bien las cosas & 44,8 & 97 \\
$\begin{array}{l}\text { Sentir que gran parte de lo que hago } \\
\text { está mal }\end{array}$ & 17,2 & 37 \\
$\begin{array}{l}\text { Creer que nada de lo que hago está } \\
\text { bien }\end{array}$ & 4,5 & 10 \\
Frecuentemente preocupado por & & \\
\hline enfermedad o dolor & 76,1 & 165 \\
\hline
\end{tabular}

En cuanto a la preocupación sobre sentir dolor o enfermar, el 76,1 \% de los niños refirió sentirse así frecuentemente (de los cuales 29,9\% mencionó sentirse siempre preocupados por el dolor y la enfermedad). Finalmente, el $16 \%$ de la muestra presentó ideación suicida. La tabla 1 muestra algunos resultados relacionados con la sintomatología depresiva.

\section{Resultados del autoconcepto}

El autoconcepto (AC) se midió por medio del cuestionario de autoconcepto general (CAG) que mide seis dimensiones: autoconcepto físico, aceptación social, autoconcepto familiar, autoconcepto intelectual, sensación de control y autoevaluación personal. Estas dimensiones se califican según tres categorías: por debajo de la norma (interpretable como deficiente auto-concepto), dentro de la norma (con una percepción apegada a la realidad) y por encima de la norma (persona con un AC altamente evaluado, posiblemente poco realista por la sobre evaluación). Los resultados del presente estudio muestran un porcentaje mayor de casos con un deficiente AC global.

A continuación, se describen de manera detallada los valores para cada factor de la escala; se destaca el porcentaje de niños con puntajes bajos en cada factor (submuestra en riesgo) y aquellos con los puntajes más altos. Se observó que el 26,3 \% de los niños presentaron dificultad para aceptar su apariencia física, en contraste con el 27,2\% de los niños que manifestaron un elevado grado de aceptación y satisfacción en su propio aspecto.

En cuanto a la percepción que los participantes tuvieron acerca de su capacidad para relacionarse con otras personas, se encontró que el 24,4\% manifestó dificultades para entablar relaciones interpersonales y solo el $17,1 \%$ se percibe con altas habilidades para ser aceptado por quienes le rodean, en otras palabras, por cada niño que tiene un alto AC social, hay dos que presentan un bajo $\mathrm{AC}$ en este factor. 
Asimismo, los resultados obtenidos muestran que un $33,6 \%$ de los niños informó sentir poca aceptación por parte de su familia; contrario a ello, el $22,1 \%$ obtuvo puntuaciones por encima de la norma, nuevamente hay más niños que manifiestan puntajes bajos, que su contraparte de alto AC familiar.

Con relación a la percepción que mostraron los niños acerca de sus capacidades intelectuales, así como su rendimiento escolar, el 30,4\% se ubicó por debajo de la norma, percibiéndose con dificultad en el ámbito académico, y un discreto $21,7 \%$ se ubica en el percentil alto de este factor, es decir, solo 2 de cada 10 niños mostraron confianza con base en sus capacidades intelectuales.

Respecto a la dimensión de autovaloración personal, se encontró que el 33,6\% de los niños se concentraron por debajo de la norma, resultado que sugiere una baja autoestima o autoevaluación en distintos aspectos de sí mismo de un tercio de la muestra. En contraste, poco más de una cuarta parte $(29 \%)$ tiene una alta autovaloración personal. De acuerdo con la escala de sensación de control, el 15,6\% percibe tener el control de su vida, mientras que el 35,9\% (más de la tercera parte) presenta dificultades para percibirse de esa manera. La figura 1 muestra algunos datos del AC del porcentaje de niños que obtuvieron puntajes más bajos y altos, según lo esperado por la norma.

\section{Relación estadísticamente significativa entre $\operatorname{sexo}$ y AC}

Al comparar la muestra por sexo se observó que los varones tienen puntajes más bajos en el autoconcepto físico (media de 29,58), con respecto a las mujeres $(31,75)$, diferencia estadísticamente significativa de acuerdo con la prueba $t$ de Student $\left(\mathrm{t}_{200}=2,43\right.$, con $\left.p<0,05\right)$. En el resto de los factores no hay diferencias estadísticamente significativas.

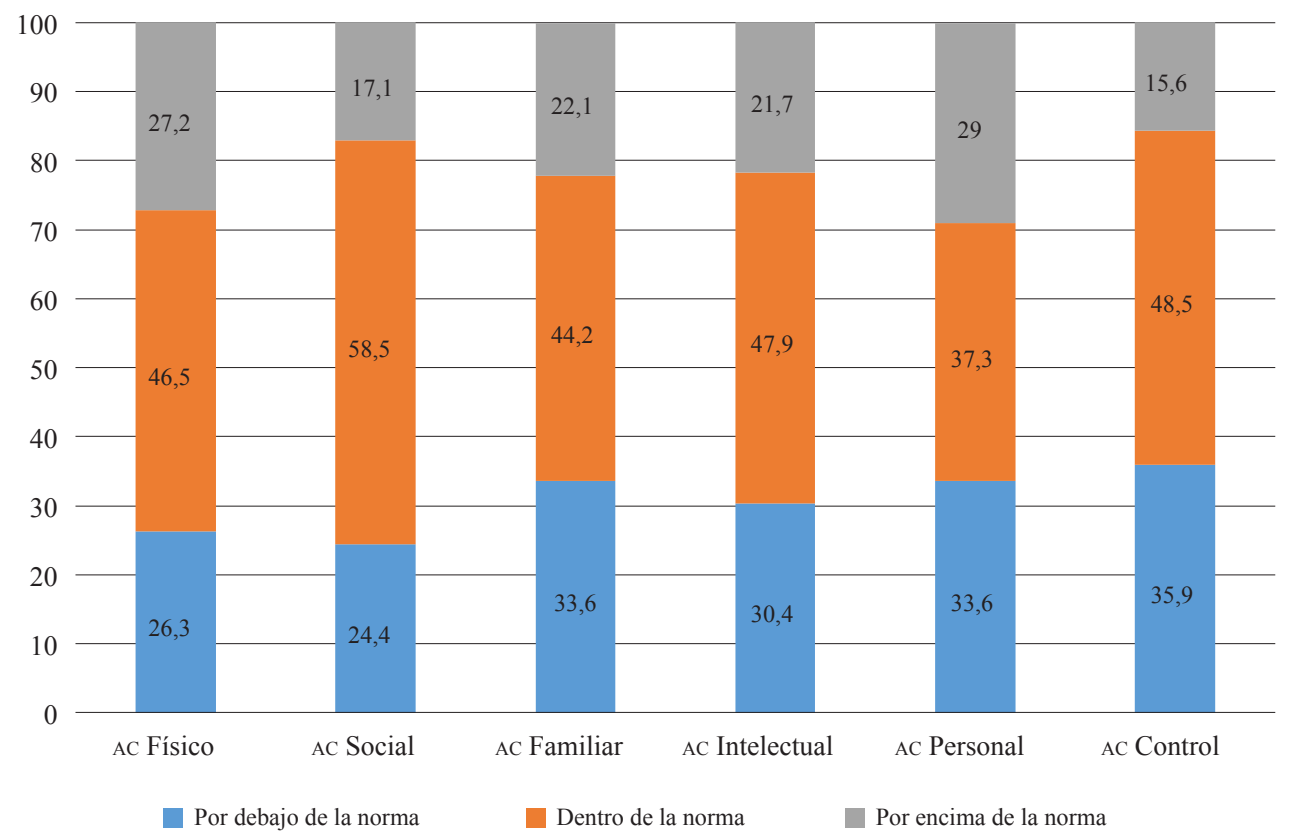

Figura 1. Porcentaje de los tres puntos de corte para cada factor del CAG

Nota: Los puntos de corte dependen de los percentiles con respecto a los siguientes rangos: de 5 a 20 por debajo de la norma; de 25 a 75 dentro de la norma y de 80 a 95 por encima de la norma. 


\section{Relación estadísticamente significativa entre grado y AC}

Al comparar la muestra de quinto y sexto grado, con los factores de autoconcepto, solamente se encontraron diferencias estadísticamente significativas con el autoconcepto control, siendo el grupo de sexto grado (media de 27,7) el que presentó la media más alta con respecto al grupo de quinto grado (media de 25,93), diferencia estadísticamente significativa de acuerdo con la prueba $t$ de Student $\left(\mathrm{t}_{198}=2,20\right.$, con $\left.p<0,05\right)$. Cabe aclarar que no se tomaron en consideración los 16 casos de cuarto grado porque el tamaño de la muestra es muy inferior al de los otros grados.

Por otro lado, también se realizaron los análisis equivalentes de prueba $t$ de Student para muestras independientes entre sexo y grado escolar con los factores de CDI y en ningún caso se encontraron diferencias estadísticamente significativas.

\section{Relación entre sintomatología depresiva y autoconcepto}

Posteriormente, se verificó la relación entre los indicadores del AC y la sintomatología depresiva. Se tomaron los puntajes absolutos de cada indicador de la escala de autoconcepto y el puntaje absoluto del CDI, para que todas las variables tuvieran un nivel de medición de tipo intervalar y se comprobó su nivel de asociación por medio del valor rho de Spearman y r de Pearson. Los resultados se pueden observar en la tabla 2.

Se observa que todos los indicadores del autoconcepto tienen un moderado grado de asociación inversamente con el puntaje total de depresión. Es decir, la sintomatología depresiva de los niños aumenta al disminuir cada uno de los elementos del autoconcepto.

De manera específica, al observar los niveles de correlación entre los factores de la sintomatología depresiva según el CDI y los factores del AC, se puede apreciar que en general el puntaje global de CDI y el factor de disforia tienen correlaciones muy similares con todos los factores del AC, mientras que las mismas correlaciones bajan ligeramente con el factor de autoestima. Esto sugiere que la disforia tiene una mayor relación con el autoconcepto que la autoestima.

\section{Resultados con la prueba estadística de regresión lineal}

Para determinar el grado de importancia de cada factor de autoconcepto sobre el puntaje total de la sintomatología depresiva, se realizó una prueba de regresión lineal por pasos sucesivos (en ambos casos los puntajes totales tienen un nivel de medición intervalar). El modelo de regresión realizado con el método de pasos sucesivos demuestra que los

Tabla 2

Grado de asociación entre los indicadores de AC y el puntaje total del CDI

\begin{tabular}{|c|c|c|c|c|c|c|c|}
\hline & & AC Físico & AC Social & AC Familiar & AC Intelectual & AC Personal & AC Control \\
\hline Puntaje CDI & Rho de Spearman & $-0,46^{* *}$ & $-0,50 * *$ & $-0,59 * *$ & $-0,52 * *$ & $-0,61 * *$ & $-0,49 * *$ \\
\hline Disforia & Rho de Spearman & $-0,43 * *$ & $-0,50 * *$ & $-0,60 * *$ & $-0,43^{* *}$ & $-0,61 * *$ & $-0,48 * *$ \\
\hline Autoestima & Rho de Spearman & $-0,41 * *$ & $-0,39 * *$ & $-0,46^{* *}$ & $-0,49 * *$ & $-0,50 * *$ & $-0,38 * *$ \\
\hline Puntaje CDI & r de Pearson & $-0,55^{* *}$ & $-0,56^{* *}$ & $-0,60 * *$ & $-0,54 * *$ & $-0,67 * *$ & $-0,52 * *$ \\
\hline Disforia & r de Pearson & $-0,52 * *$ & $-0,58 * *$ & $-0,60 * *$ & $-0,46^{* *}$ & $-0,66^{* *}$ & $-0,51 * *$ \\
\hline Autoestima & $\mathrm{r}$ de Pearson & $-0,48 * *$ & $-0,41 * *$ & $-0,48 * *$ & $-0,53 * *$ & $-0,55 * *$ & $-0,42 * *$ \\
\hline
\end{tabular}

Nota: Los ** indican un valor de correlación estadística significativa al 0,01 . 
factores del AC personal, familiar y social explica el $61,9 \%$ de la varianza total de la depresión.

Los puntajes betas fueron de $-0,37$ (para el AC personal); -0,30 (para el AC familiar); y de $-0,22$ (para el AC social). Los símbolos demuestran la relación esperada (al disminuir los puntajes de los factores del AC, aumentan el nivel de depresión).

\section{Conclusiones}

Los resultados del presente estudio arrojan información sobre los niños evaluados, que resulta relevante para ser considerada tanto en el ámbito educativo, como en la intervención clínica. Aún considerando al tamaño de la muestra como una de las limitaciones del presente estudio, fue posible detectar la presencia de sintomatología depresiva en los niños, así como algunas características del autoconcepto asociadas con esta. Cabe aclarar que se solicitó a varias escuelas primarias el poder aplicar el estudio, sin embargo, los directivos administrativos no lo permitieron aludiendo a cuestiones de posibles complicaciones con los padres de familia; en las tres escuelas que se permitió realizar la aplicación, se solicitó no mencionar sobre el tema del riesgo suicida ni con los niños ni con los padres de familia, solo hablar sobre depresión; lo anterior demuestra que todavía existen mitos y dificultades sobre la concepción de la conducta suicida en niños.

Con base en los resultados, puede concluirse que el $11,5 \%$ de la muestra total presenta algún grado de sintomatología depresiva; esta cifra está por encima de lo manifestado en otros estudios (Bernaras et al., 2011;Vogel, 2012), incluso considerando que los niños evaluados no pertenecen a un ámbito socioeconómico muy desfavorecido o marginado de la población mexicana, sino por el contrario, en su mayoría, eran estudiantes de escuelas con grupos escolares pequeños y buen nivel de atención educativa. Los síntomas mostraron puntajes de depresión severa y leve/moderada, que son predictores de riesgo suicida, lo cual también se comprueba con el alto puntaje obtenido (16\%) en ideación suicida; dicho puntaje resulta preocupante pero concordante con las estadísticas oficiales que muestran que el suicidio infantil es una realidad en alarmante incremento (INEGI, 2014; OMS, 2000). La detección oportuna de los niños con riesgo suicida, así como el adecuado abordaje de los tutores académicos y familiares, son vías importantes y adecuadas para la prevención del suicidio, pues es sabido que la conducta suicida incluye como primera fase a la ideación suicida, que posteriormente podrá devenir en tentativas suicidas o suicidio.

Los resultados del presente estudio confirman la impostergable necesidad de realizar estudios en México sobre depresión infantil, los cuales son escasos.

De manera adicional, la presente investigación describe en la muestra de niños sus niveles de autoconcepto, como lo sugiere Bragado, Hérnandez-Lloreda, Sánchez-Bernardos y Urbano (2008). Los resultados descriptivos muestran que un alto porcentaje de la muestra (aproximadamente uno de cada tres casos) presentan bajo autoconcepto familiar, personal y de sensación de control. También se encontró que la muestra de varones en general presenta niveles más bajos de autoconcepto físico que las mujeres. Esta diferencia señala un factor de riesgo entre los niños con respecto a un tema poco estudiado como lo es la parte física del autoconcepto, por lo que es importante continuar investigando sus efectos con respecto a diversos temas como, por ejemplo, el maltrato escolar (o bullying), el desempeño escolar, la resiliencia, etc.

Al correlacionar los puntajes totales de cada uno de los factores del autoconcepto con los puntajes de la sintomatología depresiva se encuentra una relación inversamente proporcional moderada de todos los factores, destacando el factor personal, familiar y social. El análisis de regresión lineal por medio del método de pasos sucesivos comprueba dicha importancia y muestra un alto porcentaje de varianza explicada $(61,9 \%)$. Los altos niveles de 
asociación del autoconcepto y su relación inversa con la sintomatología depresiva sugieren que un tratamiento preventivo que se encuentre sustentado en mejorar estas tres esferas del autoconcepto (personal, social y familiar) podría disminuir los niveles de la depresión y los problemas asociados con este como, por ejemplo, la ideación suicida. Dicha hipótesis alentadora debe ponerse a prueba para comprobar su efecto y con ello contribuir en la disminución del problema. Queda pendiente indagar las causas que provocan el bajo autoconcepto personal, de control y familiar en uno de cada tres niños de la muestra.

Los menores de edad resultan los depositarios y víctimas más vulnerables de la inadecuación del medio en el que se encuentran inmersos, así como de la falta de recursos para su óptimo desarrollo; la cobertura de elementos esenciales como la alimentación, cuidado, protección, afecto, entre otros, resultan necesarios para el adecuado crecimiento físico, social y emocional del niño. Por otro lado, las manifestaciones de un estado disfórico, triste o depresivo en un infante, no son fácilmente reconocibles por sus tutores y las personas circundantes, en ocasiones, tampoco incluso por los profesionales responsables de la tutela infantil. Esto dificulta la detección oportuna de situaciones de riesgo y señales de alarma en los niños que viven situaciones disfuncionales familiares, y que se manifestarán posteriormente con el agravamiento de síntomas $\mathrm{y}$ actos autodestructivos y/o delictivos.

Se sugiere realizar estudios sobre depresión y sus correlatos específicos en poblaciones infantiles específicas, como pueden ser en ámbitos institucionalizados, en comunidades de indígenas de alto flujo migratorio, etc. Asimismo, se recomienda utilizar en ámbitos educativos los dos instrumentos citados, para realizar evaluaciones clínicas, que deberán fortalecerse con otras estrategias diagnósticas y atender adecuadamente a los niños con signos de riesgo y sufrimiento emocional, partiendo de intervenciones psicoeducativas, tanto de prevención universal, selectiva e indicada.

\section{Referencias}

Acosta-Hernández, M. E., Mancilla-Percino, T. T., Correa-Basurto, J. J., Saavedra-Vélez, M. M., Ramos-Morales, F. R., Cruz-Sánchez, J. S., \& Duran-Niconoff, S. (2011). Depresión en la infancia y adolescencia: enfermedad de nuestro tiempo. Archivos de Neurociencias, 16(1), 20-25. Recuperado de http://www.medigraphic. com/pdfs/arcneu/ane-2011/ane111e.pdf

Asociación Americana de Psiquiatría (APA). (2014). DSM-5 Manual diagnóstico y estadístico de los trastornos mentales. Madrid: Panamericana.

Bernaras, E., Jaureguizar, J., Soroa, M., Ibabe, I., \& Cuevas, C. (2011). Child Depression in the School Context. Procedia-Social and Behavioral Sciences, 29, 198-207. doi: 10.1016/j. sbspro.2011.11.225

Borges, G., Benjet, C., Medina-Mora, M. E., Orozco, R., \& Nock, M. (2008). Suicide ideation, plan, and attempt in the Mexican adolescent mental health survey. Journal of the American Academy of Child \& Adolescent Psychiatry, 47(1), 41-52. doi: 10.1097/chi.0b013e31815896ad

Borges, G., Orozco, R., Benjet, C., \& Medina-Mora, M. E. (2010). Suicidio y conductas suicidas en México: retrospectiva y situación actual. Salud Pública de México, 52(4), 292-304. Recuperado de http://bvs.insp.mx/rsp/articulos/articulo. php?id $=002486$

Bridge, J. A., Goldstein, T. R., \& Brent, D. A. (2007). Adolescent suicide and suicidal behavior. J Child Psychol Psychiatry, 47(3), 372-394.

Chen, X., Huang, X., Wang, L., \& Chang, L. (2012). Aggression, peer relationships, and depression in Chinese children: a multiwave longitudinal study. Journal of Child Psychology and Psychiatry, 53(12), 1233-1241. doi: 10.1111/j.14697610.2012.02576.x

Cohen, J. R., Young, J. F., \& Abela, J. R. (2012). Cognitive Vulnerability to Depression in Children: An Idiographic, Longitudinal Examination of Inferential Styles. Cognitive Therapy 
and Research, 36(6), 643-654. doi: 10.1007/ s10608-011-9431-6

Del Barrio, V., \& Carrasco, M. (2004). CDI. Inventario de Depresión Infantil. Madrid: TEA Ediciones.

Esparza, N., \& Rodríguez, M. (2009). Factores contextuales del desarrollo infantil y su relación con los estados de ansiedad y depresión. Diversitas, 5(1), 47-65. Recuperado de http://revistas.usta.edu.co/index.php/diversitas/article/view/90

Gallegos, J., Linan-Thompson, S., Stark, K., \& Ruvalcaba, N. (2013). Preventing childhood anxiety and depression: Testing the effectiveness of a school-based program in Mexico. Psicología Educativa, 19(1), 37-44. doi: 10.5093/ed2013a6

Garaigordobil, M., Pérez, J. I., \& Mozaz, M. (2008). Self-concept, self-esteem and psychopathological symptoms. Psicothema, 20(1), 114-123. Recuperado de http://www.unioviedo.es/reunido/index.php/PST/article/view/8630/8494

García-Torres, B. (2001). Cuestionario de Auto-concepto General. Madrid: EOS.

Harter, S. (2006). The self. In W. Damon, R. M. Lerner \& N. Eisenberg (Eds.). Handbook of child psychology: Vol. 3. Social, emotional, and personality development (pp. 505-570). New York: Wiley.

Hipwell, A., Keenan, K., Kasza, K., Loeber, R., Stouthamer-Loeber, M., \& Bean, T. (2008). Reciprocal influences between girls' conduct problems and depression, and parental punishment and warmth: A six-year prospective analysis. Journal of Abnormal Child Psychology, 36(5), 663-677. doi: 10.1007/s10802-007-9206-4

Inguglia, C., Ingoglia, S., \& LoCoco, A. (2013). The Relation between Emotional Support, Self-concept, and Social Functioning among School-Aged Children. Bollettino Di Psicologia Applicata, 267, 3-15.

Instituto Nacional de Estadística y Geografía (INEGI). (2014). Encuesta Nacional de los Hogares 2014. Recuperado de http://www3.inegi.org.mx/sistemas/tabuladosbasicos/tabdirecto.aspx?s=es$\mathrm{t} \& \mathrm{c}=33722$
Instituto Nacional de Estadística y Geografía (INEGI). (2015a). Estadísticas a propósito del día mundial para la prevención del suicidio. Recuperado de http://www.inegi.org.mx/saladeprensa/ aproposito/2015/suicidio0.pdf

Instituto Nacional de Estadística y Geografía (INEGI). (2015b). Estadísticas de mortalidad, 2013. Consulta interactiva. Recuperado de http:// www.inegi.org.mx/est/lista_cubos/consulta. $\operatorname{asp} \mathrm{x} ? \mathrm{p}=\mathrm{adm} \& \mathrm{c}=4$

Instituto Nacional de Salud Pública (INSP). (2012). Encuesta Nacional de Salud y Nutrición 2012. Resultados nacionales. Recuperado de http:// ensanut.insp.mx/informes/ENSANUT2012ResultadosNacionales.pdf

Kovacs, M. (1992). Children's Depression Inventory Manual. Los Angeles: Western Psychological Services.

Kovacs, M. (2004). Inventario de Depresión Infantil. España: TEA Ediciones.

Maughan, B., Collishaw, S., \& Stringaris, A. (2013). Depression in Childhood and Adolescence. Journal of the Canadian Academy of Child and Adolescent Psychiatry, 22(1), 35-40. Recuperado de http://www.ncbi.nlm.nih.gov/pmc/ articles/PMC3565713/

McCabe, M., Ricciardelli, L., \& Banfield, S. (2011). Depressive Symptoms and Psychosocial Functioning in Preadolescent Children. Depression Research and Treatment, 1-7. doi: $10.1155 / 2011 / 548034$

Miranda de la Torre, I., Laborín-Álvarez, J. F., Chávez-Hernández, A. M., Sandoval, S., \& Torres, N. (2016). Propiedades psicométricas del CDI en una muestra no clínica de niños de la ciudad de Hermosillo, México. Psicología y Salud, 26(2), 273-282.

Molina, M. F., \& Raimundi, M. J. (2011). Predictores de la autoestima global en niños de escuela primaria de la Ciudad de Buenos Aires. Diferencias en función del sexo y la edad. Revista Argentina de Ciencias del Comportamiento, 
3(3), 1-7. Recuperado de http://www.revistas. unc.edu.ar/index.php/racc/article/view/5201

Organización Mundial de la Salud (OMs). (2000). The World Health Report 2000: Health Systems Improving Performance. Geneva: World Health Organization.

Organización Mundial de la Salud (oms). (2014). Prevención del suicidio. Un imperativo global. Recuperado de http://apps.who.int/iris/bitstr eam/10665/131056/1/9789241564779_eng. pdf?ua $=1 \& u a=1$

Páramo-Castillo, D., \& Chávez-Hernández, A. M. (2007). Maltrato y Suicidio Infantil en el estado de Guanajuato. Salud Mental, 30(3), 59-67.

Pérez-Olmos, I., Téllez, D., Vélez, A., \& Ibáñez-Pinilla, M. (2012). Caracterización de factores asociados con comportamiento suicida en adolescentes estudiantes de octavo grado, en tres colegios bogotanos. Revista Colombiana de Psiquiatría, 41(1), pp. 26-47.

Roberts, R. E., \& Chen, Y. W. (1995). Depressive symptoms and suicidal ideation among Mexican origin and Anglo adolescents. Journal of the American Academy of Child \& Adolescent Psychiatry, 34(1), 81-90. doi: 10.1097/00004583199501000-00018

Rodríguez-Espínola, S. (2010). Relación entre nivel socioeconómico, apoyo social percibido, género y depresión en niños. Interdisciplinaria, 27(2), 261-275. Recuperado de http://www.redalyc. org/pdf/180/18018446005.pdf

\section{Recibido: Marzo 29, 2016} Aprobado: Diciembre 14, 2016
Secretaría de Salud (SSA). (2006). Aislamiento o tranquilidad excesiva en niños, síntoma de depresión. Recuperado de http://portal.salud. gob.mx/redirector?tipo $=0 \& n \_s e c c i o n=$ Boletines\&seccion=2005-07-26_2361.html

Torre, I. M., Cubillas, M. J., Román, R., \& Valdez, E. A. (2009). Ideación suicida en población escolarizada infantil: factores psicológicos asociados. Salud Mental, 32(6), 495-502. Recuperado de http://www.inprfcd.gob.mx/pdf/ sm3206/sm3206495.pdf

Turner, H. A., Finkelhor, D., \& Ormrod, R. (2010). The effects of adolescent victimization on self-concept and depressive symptoms. Child Maltreatment, 15(1), 76-90. doi: 10.1177/1077559509349444

Vogel, W. (2012). Depression in children and adolescents. CME: Continuing Medical Education, 30(4), 114-117. Recuperado de http://search.ebscohost.com.www.e-revistas.ugto.mx/login.aspx?direct=true \&db=a9h\&AN=76476103\&lan$\mathrm{g}=\mathrm{es} \&$ site $=$ ehost-live

Yazıc1, Z., \& Taştepe, T. (2013). Relationship between family environment perception of parents and children's self-concept perception. International Journal of Human Sciences, 10(2), 98-112. Recuperado de http://j-humansciences.com/ojs/ index.php/IJHS/article/view/2487/1171 